\title{
Effect of plasma response on the fast ion losses due to ELM control coils in ITER
}

\author{
Jari Varje ${ }^{1}$, Otto Asunta ${ }^{1,2}$, Mario Cavinato ${ }^{3}$, Mario Gagliardi ${ }^{3}$, \\ Eero Hirvijoki ${ }^{1,4}$, Tuomas Koskela ${ }^{1}$, Taina Kurki-Suonio ${ }^{1}$, \\ Yueqiang Liu ${ }^{5}$, Vassili Parail ${ }^{5}$, Gabriella Saibene ${ }^{3}$, Seppo Sipilä ${ }^{1}$, \\ Antti Snicker ${ }^{1,6}$, Konsta Särkimäki ${ }^{1}$, Simppa Äkäslompolo ${ }^{1}$
}

\footnotetext{
${ }^{1}$ Department of Applied Physics, Aalto University, FI-00076 AALTO, Finland ${ }^{2}$ Tokamak Energy Ltd, 120A Olympic Avenue, Milton Park, Oxfordshire, OX14 45A, United Kingdom

${ }^{3}$ Fusion for Energy, Torres Diagonal Litoral, Edificio B3, 08019 Barcelona, Spain ${ }^{4}$ Department of Applied Physics, Chalmers University of Technology, Gothenburg, Sweden

${ }^{5}$ Culham Centre for Fusion Energy, Culham Science Center, Abingdon, Oxfordshire, OX14 3DB, United Kingdom

${ }^{6}$ Max-Planck-Institut für Plasmaphysik, Garching, Germany

E-mail: jari.varje@aalto.fi
}

15 February 2016

\begin{abstract}
Mitigating edge localized modes (ELMs) with resonant magnetic perturbations (RMPs) can increase energetic particle losses and resulting wall loads, which have previously been studied in the vacuum approximation. This paper presents recent results of fusion alpha and NBI ion losses in the ITER baseline scenario modelled with the Monte Carlo orbit following code ASCOT in a realistic magnetic field including the effect of the plasma response. The response was found to reduce alpha particle losses but increase NBI losses, with up to $4.2 \%$ of the injected power being lost. Additionally, some of the load in the divertor was found to be shifted away from the target plates toward the divertor dome.
\end{abstract}




\section{Introduction}

Operating a high performance plasma in the H-mode normally results in edge localized modes (ELMs) which are violent relaxation processes of the edge pedestal region that rapidly deposit large amounts of energy in the divertor. The high power loads from ELMs are expected to be the primary driver for the erosion of the target plates in ITER, limiting the lifetime of the divertor cassettes [1]. Thus suppression or mitigation of ELMs is a critical part of the design and operation of ITER. One of the leading mitigation methods is the application of resonant magnetic perturbations (RMPs) on the plasma using an array of in-vessel ELM control coils (ECCs) [2]. While RMPs reduce the severity of the ELMs, the non-axisymmetric perturbations also reduce the confinement of energetic particles, resulting in increased fast ion losses and wall loads, both on the divertor and the blanket.

Results from experiments in DIII-D have yielded criteria associating the vacuum magnetic perturbation to ELM mitigation effectiveness [3]. The width of the region with overlapping magnetic islands, corresponding to the region of perturbation-induced stochasticity, should extend sufficiently far in from the separatrix. This criteria has further been linked to the formation of a transportenhancing island on top of the edge pedestal [4]. The DIII-D criteria have been applied to ITER, and sufficient coil currents for ELM mitigation have been determined for several ITER operating scenarios [5].

The effect of ECCs on fast ion losses has previously been modelled in the vacuum approximation $[6,7,8,9]$, where it was presumed that plasma would respond to the applied RMP field by dampening the penetration of the perturbations and reducing losses. However, recent simulation results [10] suggest this is not generally the case. The plasma response can increase stochasticity near the edge, even while hindering island formation deeper inside the plasma. Also of interest is the effect on the distribution of losses, where the response may diffuse or concentrate the particle flux on the plasma facing components, possibly resulting in hot spots with high peak loads.

In this paper, the effect of ECCs and plasma response on fusion alpha and NBI ion losses in ITER 15 MA Q=10 baseline scenario is studied in the presence of magnetic perturbations from ferritic components.

\section{Model and methods}

The simulations of the wall loads in this study extend the methods previously applied to the 15 MA baseline case [11]. The particles were followed using the Monte Carlo orbit-following code ASCOT [12] in a realistic 3D magnetic field, including perturbations caused by the ripple-mitigating ferritic inserts and three pairs of European test blanket modules, which also contain ferritic material.

The plasma equilibrium and profiles (Figure 1) were prepared using JINTRAC [13]. The plasma profiles in the baseline scenario still include carbon as an impurity species, but its effect on fast ion losses has been found negligible [11]. The fusion alpha test particles were initialized uniformly in the plasma volume and weighted according to thermal fusion reactivity at their birthplace. Total fusion alpha power in the baseline scenario was $85 \mathrm{MW}$. NBI ions were generated for the two ITER injectors in both on-axis and off-axis configurations 

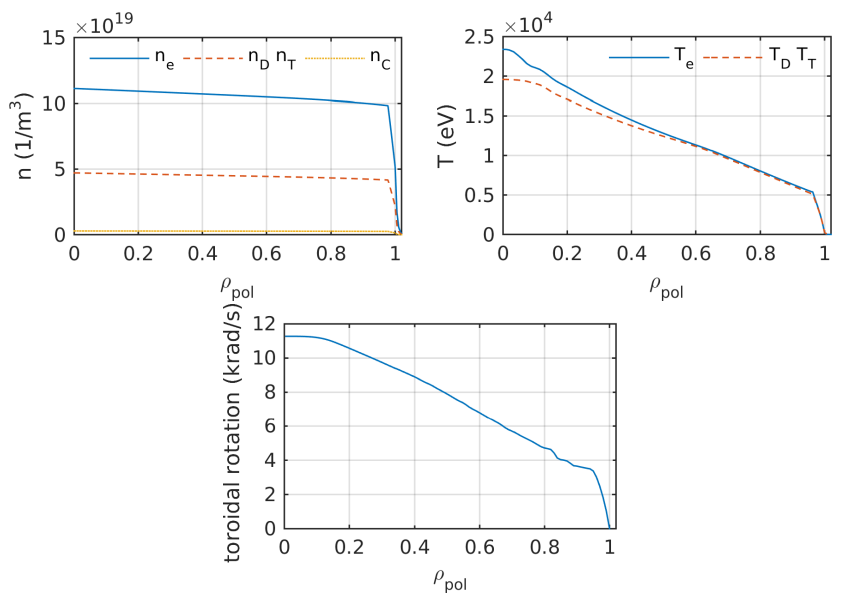

Figure 1: Electron, ion and carbon impurity densities (top left), electron and ion temperatures (top right) and toroidal plasma rotation velocity (bottom) in the 15MA baseline scenario.
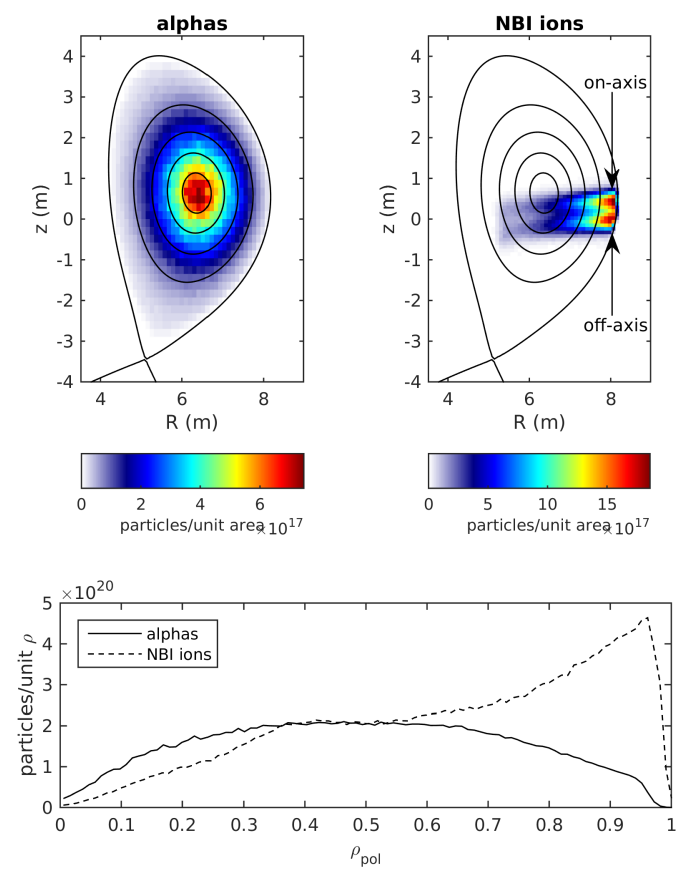

Figure 2: Initial distributions for the fusion alphas and NBI ions in the poloidal cross section (top) and as a function of the radial coordinate $\rho_{\text {pol }}=$ $\sqrt{\left(\psi-\psi_{\text {axis }}\right) /\left(\psi_{\text {sep }}-\psi_{\text {axis }}\right)}$ (bottom). 


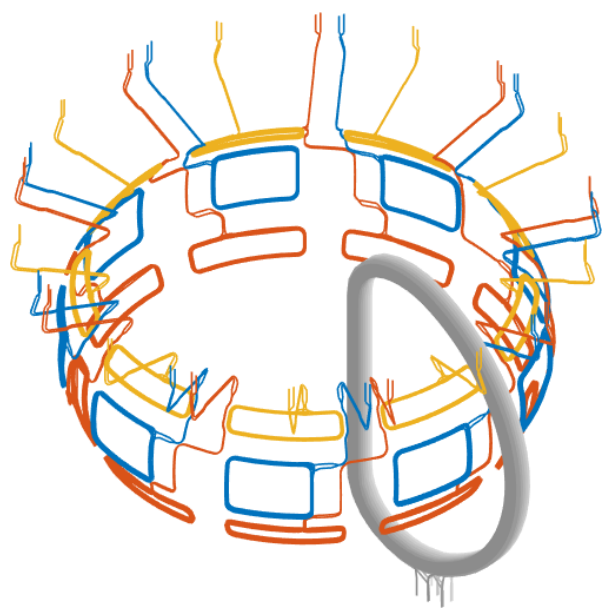

Figure 3: Upper, equatorial and lower ELM control coils and their current leads used in the calculation of the RMP field, together with an example of the TF coils.

with a total NBI heating power of $33 \mathrm{MW}$. An ensemble of 100000 test particles was used when estimating the total losses for fusion alphas and the NBI ions in both configurations (Figure 2). An ensemble of 300000 NBI test particles were used in the simulation for the peak heat load estimates.

The magnetic perturbations caused by the ferritic components were computed with the finite-element solver COMSOL [14]. In the first step, magnetization of the components was computed from the magnetic fields produced by the poloidal and toroidal coils and the plasma current. In the second step, the magnetic perturbations resulting from the magnetization were computed and added to the equilibrium magnetic field together with the toroidal field ripple. The RMP field produced by the ECCs was computed from a detailed coil geometry (Figure 3) using the Biot-Savart integrator BioSaw [8]. The coil currents were set according to the reference case for a $4.5 \mathrm{keV}$ pedestal temperature baseline plasma, with an $\mathrm{n}=3$ mode $45 \mathrm{kAt}$ maximum current and phase shifts of $86^{\circ}, 0^{\circ}$ and $34^{\circ}$ for the upper, equatorial and lower coil rows respectively [5].

The response of the plasma to the magnetic perturbations was computed using the resistive MHD code MARS-F [15]. The MARS-F model has been extensively benchmarked and validated against other models and experiments for the plasma response computations $[16,17,18]$. Response in the toroidal modes $n=1$ through $n=6$ was included, as the contribution from the higher $n$ modes was found to be negligible. The original vacuum approximation was used for the higher modes. As shown in the Poincaré plots in Figure 4, the effect of the plasma response is to significantly reduce the width of the islands near the edge of the plasma. However, the response increases the stochasticity close to the edge $\left(\rho_{\text {pol }}>0.95\right)$, resulting in field lines being rapidly lost to the wall. A shift of approximately 60-70 degrees in the toroidal island location can be seen, which is consistent with results in a similar study for ASDEX Upgrade [19]. Additionally, the plasma response shifts the location of the separatrix, evident in the displacement of the X-point by up to $5 \mathrm{~mm}$ (Figure 5 ).

To accurately determine the particle strike points on the first wall, a detailed 

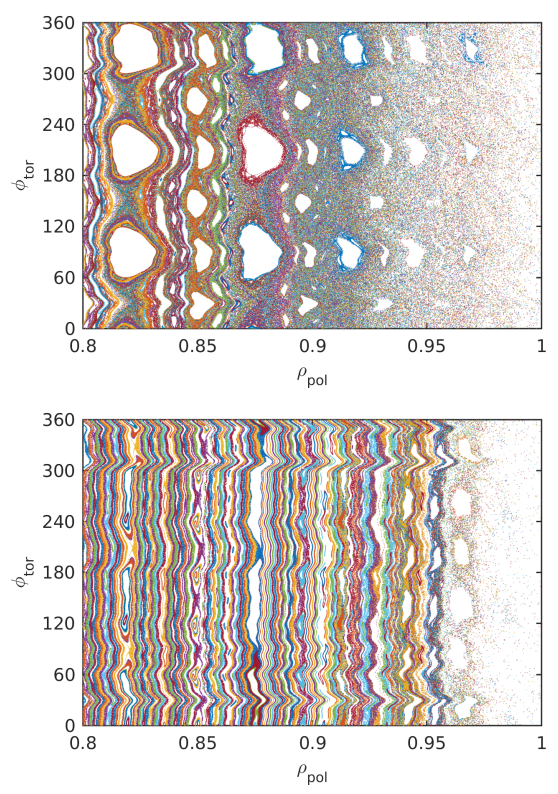

Figure 4: Poincaré plots of the magnetic field line structure close to the last closed flux surface on the outer midplane without (top) and with plasma response (bottom). Field lines were followed from the outer midplane until they intersected the wall or completed 5000 toroidal orbits.

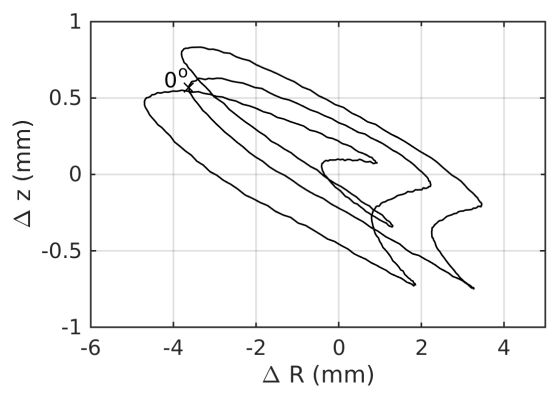

Figure 5: Displacement of the X-point, as determined by locating the local minimum of the poloidal magnetic field, in the plasma response case with respect to the vacuum approximation. The plot traces the displacement around the plasma in the toroidal direction. 
Table 1: Total fast ion losses, with non-ECC results from [11]. Errors from the Monte Carlo method were estimated using bootstrapping.

$$
\text { No ECC With ECC }
$$

\begin{tabular}{lrrr} 
& & Vacuum & Response \\
\hline Alphas & & & \\
Wall & $40 \mathrm{~kW}$ & $70 \pm 4 \mathrm{~kW}$ & $157 \pm 5 \mathrm{~kW}$ \\
Divertor & $130 \mathrm{~kW}$ & $1925 \pm 15 \mathrm{~kW}$ & $1343 \pm 12 \mathrm{~kW}$ \\
NBI ions & (on-axis) & & \\
Wall & $7 \mathrm{~kW}$ & $3 \pm 1 \mathrm{~kW}$ & $3 \pm 1 \mathrm{~kW}$ \\
Divertor & $1 \mathrm{~kW}$ & $1273 \pm 20 \mathrm{~kW}$ & $1381 \pm 27 \mathrm{~kW}$ \\
NBI ions & (off-axis) & & \\
Wall & $7 \mathrm{~kW}$ & $6 \pm 1 \mathrm{~kW}$ & $7 \pm 1 \mathrm{~kW}$ \\
Divertor & $1 \mathrm{~kW}$ & $1026 \pm 17 \mathrm{~kW}$ & $1177 \pm 23 \mathrm{~kW}$
\end{tabular}
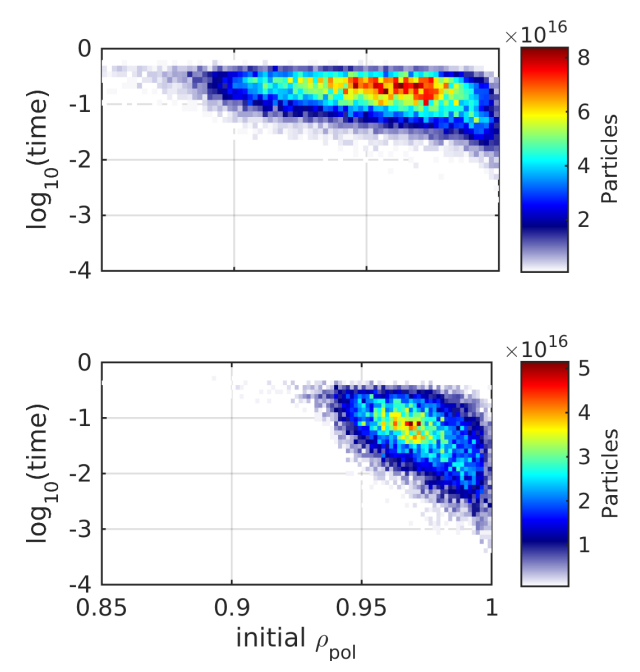

Figure 6: Distribution of lost NBI particles as a function of the radial coordinate $\rho_{\text {pol }}$ of their birthplace and the time to reach the wall in the vacuum approximation (top) and with plasma response (bottom).

3D wall model was used in the simulations. The wall model is based on a CAD design of the first wall and divertor, which were simplified using a ray-tracing method and smoothing to approximately 300000 triangles with a mean size of $25 \mathrm{~cm}^{2}$. To characterize the losses, the first wall and the divertor were distinguished in the wall model. The divertor was further divided into the inner and outer target plates, the dome covering the center part of the divertor, and the structures below the dome.

\section{Fast ion losses}

Both the fusion alpha particles as well as NBI ions have previously been found to be well confined in the baseline scenario without ECCs [11]. Including the perturbations from the ECCs has negligible effect on the energetic particle losses 
Table 2: Fast ion losses to the divertor separated by strike point location. Monte Carlo errors were estimated using the bootstrapping method.

Inner target Outer target Divertor dome Below dome

\begin{tabular}{lrrrr}
\hline Alphas & & & & \\
Without response & $825 \pm 9 \mathrm{~kW}$ & $877 \pm 10 \mathrm{~kW}$ & $205 \pm 5 \mathrm{~kW}$ & $18 \pm 1 \mathrm{~kW}$ \\
With response & $542 \pm 7 \mathrm{~kW}$ & $386 \pm 7 \mathrm{~kW}$ & $349 \pm 7 \mathrm{~kW}$ & $66 \pm 3 \mathrm{~kW}$ \\
NBI ions (on-axis) & & & & \\
Without response & $795 \pm 15 \mathrm{~kW}$ & $238 \pm 8 \mathrm{~kW}$ & $188 \pm 7 \mathrm{~kW}$ & $52 \pm 4 \mathrm{~kW}$ \\
With response & $271 \pm 11 \mathrm{~kW}$ & $143 \pm 8 \mathrm{~kW}$ & $846 \pm 21 \mathrm{~kW}$ & $121 \pm 8 \mathrm{~kW}$ \\
NBI ions (off-axis) & & & \\
Without response & $597 \pm 14 \mathrm{~kW}$ & $220 \pm 7 \mathrm{~kW}$ & $173 \pm 7 \mathrm{~kW}$ & $35 \pm 3 \mathrm{~kW}$ \\
With response & $166 \pm 7 \mathrm{~kW}$ & $199 \pm 9 \mathrm{~kW}$ & $737 \pm 19 \mathrm{~kW}$ & $74 \pm 5 \mathrm{~kW}$
\end{tabular}
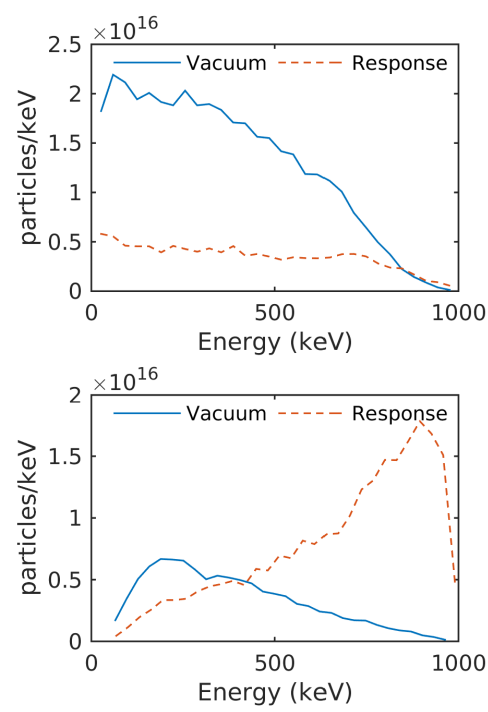

Figure 7: Energy distribution of the particles impacting the inner target (top) and divertor dome (bottom) in vacuum approximation and with plasma response. 

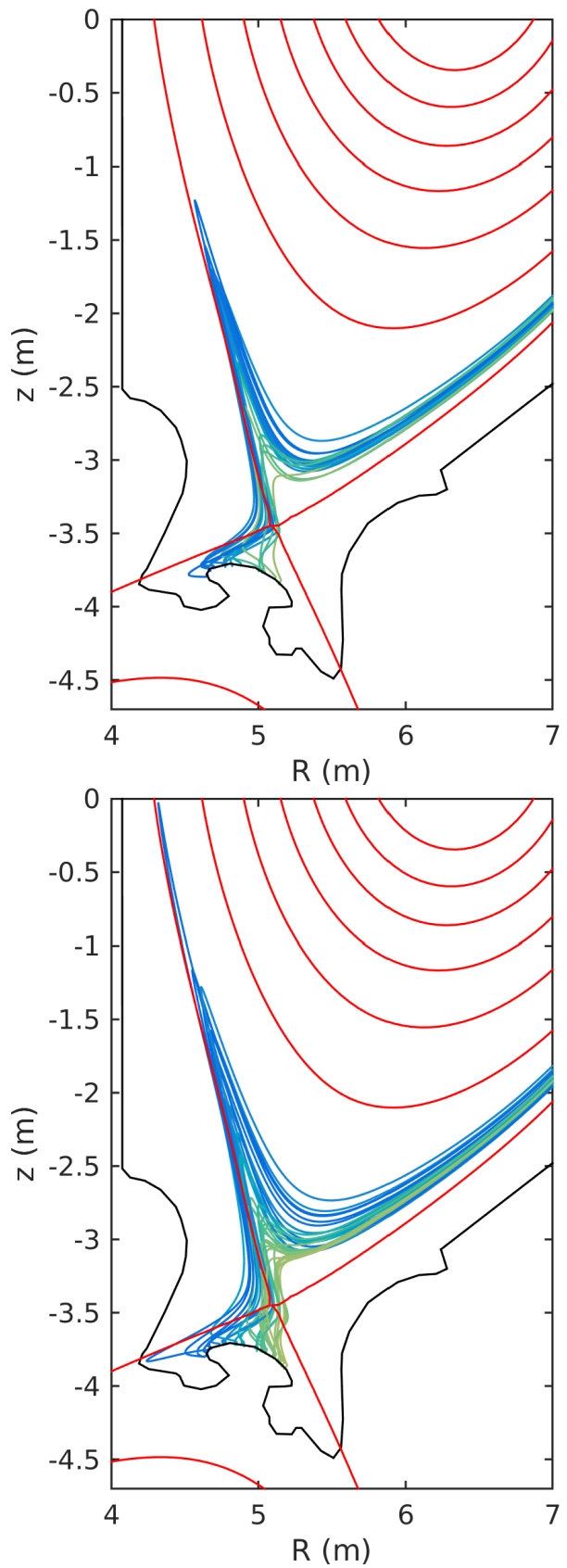

Figure 8: Typical trajectories of particles hitting the dome without plasma response (top) and with plasma response (bottom). Colour is used to distinguish particles with different strike points across the dome. 


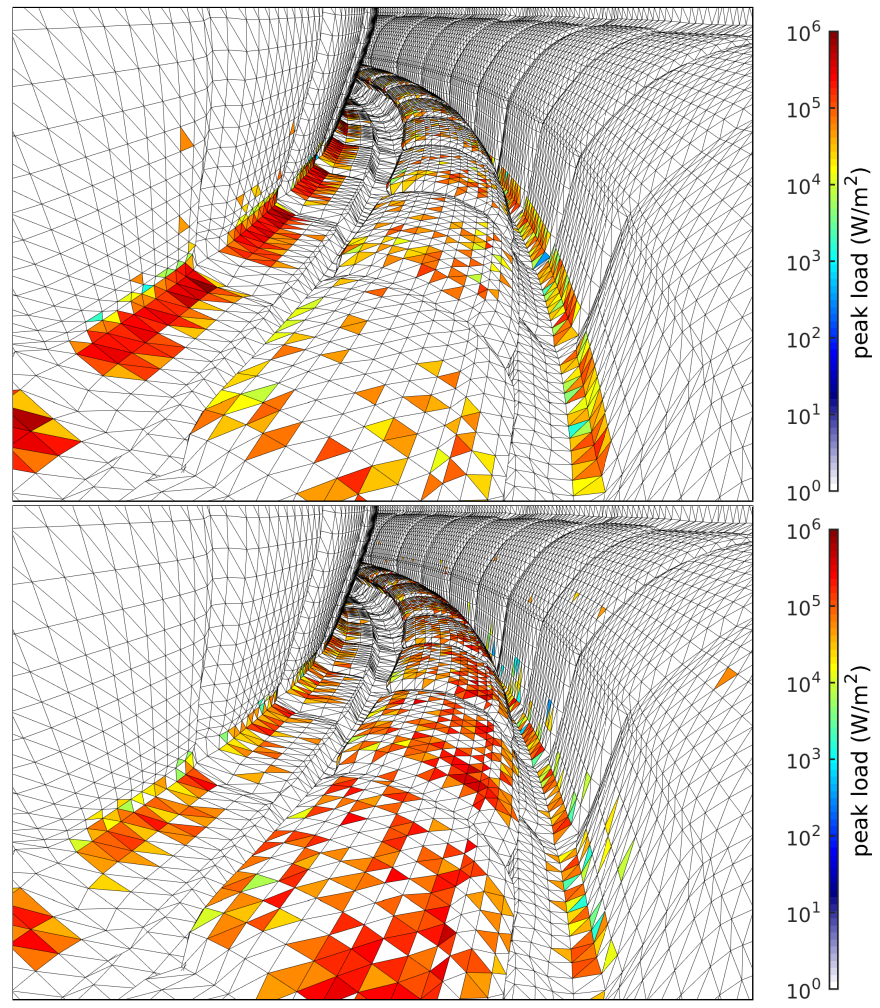

Figure 9: Peak loads due to NBI ions on the divertor targets and dome without plasma response (top) and with plasma response (bottom). Losses were also observed on elements on the underside of the dome. 
to the wall (Table 1). The losses to the divertor increase dramatically, as the ECC-enhanced stochasticity rapidly depletes the particles produced or ionized near the edge.

Including the plasma response reduces fusion alpha power loss by $25 \%$ due to the shielding effect in $\rho_{\text {pol }}<0.95$, as few alphas are born further out. However, the NBI losses increase by $8-15 \%$ with the plasma response, since their birth profile is peaked closer to the plasma edge where the stochasticity is enhanced. For the alpha particles, the losses with plasma response represent $1.8 \%$ of the total fusion alpha power. The NBI ion losses in the off- and on-axis configurations represent $3.6 \%$ and $4.2 \%$ of the total injected NBI power, respectively. The difference between the on-axis and off-axis losses is due to the off-axis injection trajectory being located below the midplane, where the particles can reach further inwards compared to the on-axis configuration.

In the vacuum approximation, the lost NBI particles originate primarily from $\rho_{\text {pol }}>0.9$, while in the plasma response case the losses are cocentrated to the region $\rho_{\text {pol }}>0.94$ (Figure 6 ). These values closely correspond with the width of the stochastic region in Figure 4. Additionally, the lost particles reach the wall faster in the plasma response case, which is consistent with the shorter connection lengths of the field lines close to the edge.

Without plasma response, $80 \%$ of the power loss from NBI ions impinges on the target plates (Table 2). Upon application of the plasma response, the load is shifted towards the divertor dome, which then receives $62 \%$ of the losses.

While the energy distribution of the particles reaching the inner target is similar in the vacuum approximation and plasma response, the energy of the particles impacting the divertor dome is significantly higher with the response (Figure 7). This is consistent with the faster losses in the plasma response case (Figure 6), since the particles do not have the time to slow down through collisions. Thus it would appear the shift in losses is caused by particles on slightly wider banana orbits due to their higher energy in the plasma response case (Figure 8).

The peak fluxes in the divertor are approximately $1 \mathrm{MW} / \mathrm{m}^{2}$ in both cases (Figure 9), which is within the design limits both for the targets as well as the divertor dome [20]. However, a significant heat load of up to $121 \mathrm{~kW}$ impinges on the underside of the dome.

\section{Discussion}

Based on the simulation results, it is apparent that the effect of the plasma response cannot simply be assumed to be favourable, but it depends on the operating scenario, plasma profiles and the source of energetic particles. While the effect on the alpha particle losses is benign, the NBI losses approach a significant fraction of the total NBI heating power. The losses are less than predicted in previous studies where full $90 \mathrm{kAt}$ coil current was applied, but even the case presented here can noticeably reduce the available heating power margin and possibly limit the plasma performance. Redistribution of the losses due to the plasma response can also expose poorly protected areas to heating from fast ions, risking damage to the structures.

While including the effect of plasma response represents a step forward in the fidelity of ECC-induced fast ion loss analysis, further study is still called for. 
With plasma response, the shielding and amplification of the perturbations is represented in the magnetic field structure, but the equilibrium and the plasma profiles are still modelled in the vacuum approximation. The lost field lines near the edge likely cannot support as steep pedestal profiles as those resulting from the vacuum approximation. The lower density near the edge would reduce both the fusion yield as well as neutral beam ionization near the edge, likely resulting in fewer losses. A self-consistent plasma equilibrium, including the effect of the perturbations and the plasma response, would thus be required.

With the level of detail in the wall model and the number of test particles used in the presented simulations, the peak loads on the divertor target plates can be estimated with good confidence. Modelling the peak heat load on the structures under the divertor dome, however, would require more detailed wall geometry that includes the cooling systems and support structures, to identify any localized heating that could damage the components.

\section{Acknowledgements}

This work was partially funded by Fusion For Energy Grant 379 and the Academy of Finland project No. 259675, and has also received funding from Tekes - the Finnish Funding Agency for Innovation under the FinnFusion Consortium. The work was carried out using the HELIOS supercomputer system at International Fusion Energy Research Centre, Aomori, Japan, under the Broader Approach collaboration between Euratom and Japan, implemented by Fusion for Energy and JAEA. The supercomputing resources of CSC - IT center for science were utilised in the studies. Some of the calculations were performed using computer resources within the Aalto University School of Science "Science-IT" project.

\section{References}

[1] PR Thomas et al. ELM physics and ELM mitigation in ITER. In Fusion Energy 2008 (Proc. 22nd Int. Conf. Geneva, 2008) (Vienna: IAEA), 2008.

[2] A Loarte, G Huijsmans, S Futatani, LR Baylor, TE Evans, DM Orlov, O Schmitz, M Becoulet, P Cahyna, Y Gribov, et al. Progress on the application of ELM control schemes to ITER scenarios from the non-active phase to DT operation. Nuclear Fusion, 54(3):033007, 2014.

[3] ME Fenstermacher, TE Evans, TH Osborne, MJ Schaffer, MP Aldan, P Gohil, I Joseph, RA Moyer, PB Snyder, RJ Groebner, et al. Effect of island overlap on edge localized mode suppression by resonant magnetic perturbations in DIII-D. Physics of Plasmas (1994-present), 15(5):056122, 2008.

[4] MR Wade, R Nazikian, TE Evans, NM Ferraro, RA Moyer, DM Orlov, RJ Buttery, ME Fenstermacher, AM Garofalo, MA Lanctot, et al. Advances in the physics understanding of ELM suppression using resonant magnetic perturbations in DIII-D. Nuclear Fusion, 55(2):023002, 2015.

[5] T.E. Evans, D.M. Orlov, A. Wingen, W. Wu, A. Loarte, T.A. Casper, O. Schmitz, G. Saibene, M.J. Schaffer, and E. Daly. 3D vacuum magnetic 
field modelling of the ITER ELM control coil during standard operating scenarios. Nuclear Fusion, 53(9):093029, 2013.

[6] K. Shinohara, T. Kurki-Suonio, D. Spong, O. Asunta, K. Tani, E. Strumberger, S. Briguglio, T. Koskela, G. Vlad, S. Günter, G. Kramer, S. Putvinski, K. Hamamatsu, and ITPA Topical Group on Energetic Particles. Effects of complex symmetry-breakings on alpha particle power loads on first wall structures and equilibrium in ITER. Nuclear Fusion, 51(6):063028, 2011.

[7] K. Tani, K. Shinohara, T. Oikawa, H. Tsutsui, S. Miyamoto, Y. Kusama, and T. Sugie. Effects of elm mitigation coils on energetic particle confinement in iter steady-state operation. Nuclear Fusion, 52(1):013012, 2012.

[8] T. Koskela, O. Asunta, E. Hirvijoki, T. Kurki-Suonio, and S. Äkäslompolo. ITER edge-localized modes control coils: the effect on fast ion losses and edge confinement properties. Plasma Physics and Controlled Fusion, 54(10):105008, 2012.

[9] Taina Kurki-Suonio, Simppa Äkäslompolo, Konsta Särkimäki, Seppo Sipilä, Antti Snicker, Eero Hirvijoki, Otto Asunta, Tuomas Koskela, and Mario Gagliardi. ITER energetic particle confinement in the presence of ELM control coils and european tbms. In 25th Fusion Energy Conference (FEC 2014) Saint Petersburg, Russia 13 -18 October 2014, pages TH/P3P30. IAEA, 2014.

[10] D Pfefferlé, C Misev, WA Cooper, and JP Graves. Impact of RMP magnetic field simulation models on fast ion losses. Nuclear Fusion, 55(1):012001, 2015.

[11] Simppa Äkäslompolo, Taina Kurki-Suonio, Otto Asunta, Mario Cavinato, Mario Gagliardi, Eero Hirvijoki, Gabriella Saibene, Seppo Sipilä, Antti Snicker, Konsta Särkimäki, and Jari Varje. ITER fast ion confinement in the presence of the european test blanket module. Nuclear Fusion, 55(9):093010, 2015.

[12] E. Hirvijoki, O. Asunta, T. Koskela, T. Kurki-Suonio, J. Miettunen, S. Sipilä, A. Snicker, and S. Äkäslompolo. ASCOT: Solving the kinetic equation of minority particle species in tokamak plasmas. Computer Physics Communications, 185(4):1310-1321, 2014.

[13] V. Parail, R. Albanese, R. Ambrosino, J.-F. Artaud, K. Besseghir, M. Cavinato, G. Corrigan, J. Garcia, L. Garzotti, Y. Gribov, F. Imbeaux, F. Koechl, C.V. Labate, J. Lister, X. Litaudon, A. Loarte, P. Maget, M. Mattei, D. McDonald, E. Nardon, G. Saibene, R. Sartori, and J. Urban. Self-consistent simulation of plasma scenarios for ITER using a combination of $1.5 \mathrm{D}$ transport codes and free-boundary equilibrium codes. Nuclear Fusion, 53(11):113002, 2013.

[14] S Äkäslompolo, O Asunta, T Bergmans, M Gagliardi, J Galabert, E Hirvijoki, T Kurki-Suonio, S Sipilä, A Snicker, and K Särkimäki. Calculating the 3D magnetic field of ITER for european TBM studies. Fusion Engineering and Design, 98-99:1039 - 1043, 2015. Proceedings of the 28th Symposium On Fusion Technology (SOFT-28). 
[15] Y. Q. Liu, A. Bondeson, C. M. Fransson, B. Lennartson, and C. Breitholtz. Feedback stabilization of nonaxisymmetric resistive wall modes in tokamaks. I. electromagnetic model. Physics of Plasmas (1994-present), $7(9): 3681-3690,2000$

[16] Yueqiang Liu, A Kirk, Y Gribov, MP Gryaznevich, TC Hender, and E Nardon. Modelling of plasma response to resonant magnetic perturbation fields in MAST and ITER. Nuclear Fusion, 51(8):083002, 2011.

[17] MJ Lanctot, H Reimerdes, AM Garofalo, MS Chu, JM Hanson, YQ Liu, GA Navratil, IN Bogatu, Y In, GL Jackson, et al. Measurement and modeling of three-dimensional equilibria in DIII-D. Physics of Plasmas (1994present), 18(5):056121, 2011.

[18] JD King, EJ Strait, SA Lazerson, NM Ferraro, NC Logan, SR Haskey, J-K Park, JM Hanson, MJ Lanctot, Yueqiang Liu, et al. Experimental tests of linear and nonlinear three-dimensional equilibrium models in DIII-D. Physics of Plasmas (1994-present), 22(7):072501, 2015.

[19] A Kirk, W Suttrop, IT Chapman, Yueqiang Liu, R Scannell, AJ Thornton, L Barrera Orte, P Cahyna, T Eich, R Fischer, et al. Effect of resonant magnetic perturbations on low collisionality discharges in MAST and a comparison with ASDEX Upgrade. Nuclear Fusion, 55(4):043011, 2015.

[20] R.A. Pitts, S. Carpentier, F. Escourbiac, T. Hirai, V. Komarov, A.S. Kukushkin, S. Lisgo, A. Loarte, M. Merola, R. Mitteau, A.R. Raffray, M. Shimada, and P.C. Stangeby. Physics basis and design of the ITER plasma-facing components. Journal of Nuclear Materials, 415(1, Supplement):S957-S964, 2011. Proceedings of the 19th International Conference on Plasma-Surface Interactions in Controlled Fusion. 